GRADUATE THESIS REPORT

\title{
Design and development of a wearable system for the analysis of respiratory patterns for health monitoring in seniors
}

\author{
Oscar Eduardo Castrejón Mejía, Pedro C. Santana- \\ Mancilla, Silvia B. Fajardo-Flores
}

Published: 30 November 2021

\begin{abstract}
Wearable technology for non-invasive breathing monitoring has shown positive results in clinical tests for the early detection of more severe diseases. Due to the above, this project proposes to predict respiratory diseases by applying Artificial Intelligence techniques to the data obtained from the respiratory rate to identify risks to people's health through a non-invasive wearable device. User-centered methods are being used to design a prototype informed by the needs and characteristics of the users.
\end{abstract}

\section{Keywords:}

Co-design; Older adults; Remote health monitoring; Machine learning; Internet of Medical Things.

\section{Introducción}

Actualmente, podemos darnos cuenta de que la tecnología está relacionada con la mayoría de las cosas que vemos y hacemos en nuestras actividades cotidianas, lo que genera que cada vez existan más proyectos de apoyo para actividades del día a día mediadas por tecnología. El enfoque de este proyecto está dirigido para adultos mayores de 60 años, un grupo poblacional que es vulnerable en aspectos de salud, ya que, de este grupo, el $26,3 \%$ no cuenta con seguro social de salud y se encuentran vulnerables en los temas de compañía y cuidados, esto se debe a los cambios estructurales que sufren las familias [7].

La población de adultos mayores de 60 años alcanzó la cifra de 200 millones en 1950, y desde 1975 ha ido en aumento hasta superar los 350 millones. En el año 2000 se calcularon mediante proyecciones demográficas alrededor de 600 millones, se estima que esta cifra será duplicada en 2025 [10].

En adultos mayores, un reposo en cama de tiempo prolongado acompañado de poca o nula práctica de actividad física previa

Castrejón Mejía, Oscar. Eduardo., Santana-Mancilla, Pedro. C., FajardoFlores, Silvia. B.

IHCLab

Facultad de Telemática

Universidad de Colima

ocastrejon@ucol.mx,psantana@ucol.mx,medusa@ucol.mx puede provocar que se desarrollen enfermedades relacionadas con los sistemas cardiovascular, respiratorio y músculo esquelético, lo que también puede provocar que se agraven [6]. Es por eso, que la recuperación ante un padecimiento es más lenta en comparación con una persona más joven ya que, por ejemplo, un adulto de entre 60 y 79 años puede presentar un deterioro funcional de hasta $33 \%$ en una o más actividades del día a día mientras que este porcentaje aumenta a casi el 50\% al llegar a los 80 años o más.[6].

Uno de los padecimientos más preocupantes son las infecciones de vías respiratorias bajas (IVRB), ya que son las causantes a nivel mundial de una alta mortalidad en adultos. Dentro de las IVRB se encuentra la neumonía o la Enfermedad Pulmonar Obstructiva Crónica, las cuales constituyen una fuerte amenaza hacia la salud de los adultos [3]. El interés en esta área se debe también a la emergencia sanitaria generada por la enfermedad COVID-19 que ha provocado que las enfermedades respiratorias sean clasificadas como la enfermedad transmisible más mortal a nivel mundial. Esto es de especial importancia en la atención de personas mayores, ya que las enfermedades respiratorias son más mortales en esta población y pueden requerir mayores tiempos de hospitalización.

Con la emergencia sanitaria, a nivel mundial los hospitales y clínicas tienen espacios muy limitados, albergar a pacientes con estancias prolongadas es complicado y de costos elevados. El desarrollo de modelos para la atención y cuidado de pacientes en su propio domicilio es de suma importancia ya que así se puede proporcionar una atención que mejora la calidad de vida tanto psicológica y física sin tener el desgaste de traslados y estadías hospitalarias incluyendo los altos gastos médicos [1].

Teniendo en cuenta esto, se plantea recurrir al Internet de las Cosas Médicas (IoMT, por sus siglas en inglés, Internet of Medical Things), que es la interconexión de varios instrumentos, dispositivos o sensores médicos a Internet, los que tendrán la funcionalidad de recolectar la información del usuario por medio de sensores con el objetivo de conocer en tiempo real el estado físico de los usuarios y realizar un diagnóstico con cierto grado de precisión [11].

En conjunto con la tecnología IoMT, se tendrá que incorporar un modelo de Aprendizaje Automático (ML, por sus siglas en inglés, Machine Learning), mismo que consiste en generar programas informáticos utilizando datos históricos con el propósito de ser autónomo, podrá ser capaz de resolver un problema de 
manera eficiente mediante su propio conocimiento [8]. Con la intersección de estas tecnologías, se puede obtener un sistema completo que puede funcionar de manera remota para el monitoreo de la salud en los adultos mayores. La implementación de este sistema IoMT + ML será a través de un dispositivo vestible no invasivo, para que tenga mayores posibilidades de ser aceptado por los adultos mayores.

Es importante mencionar que el alcance de este proyecto es su diseño y desarrollo centrados en el usuario y la aceptación tecnológica por parte de los usuarios. En una segunda etapa (fuera del alcance de este proyecto) se realizará la validación médica del sistema desarrollado en este proyecto.

\section{Motivación de la investigación}

La tecnología vestible para la monitorización respiratoria no invasiva ha mostrado resultados clínicos positivos para la detección precoz de una patología más grave.

Es por lo que se busca crear este sistema, tomando en cuenta que el uso de la tecnología hoy en día es una alternativa viable y eficaz para desarrollar soluciones cada día más sofisticadas y complejas. Éste podría ayudar a disminuir hospitalizaciones o muertes por causas de enfermedades respiratorias severas principalmente en adultos mayores en caso de que se utilice de manera adecuada.

\section{Trabajos relacionados}

La revisión de literatura dio como resultado el uso de técnicas de aprendizaje automático para analizar la información de los pacientes y realizar diagnósticos con base en esos datos, así como el uso de aplicaciones móviles para el monitoreo de los pacientes.

Un sistema de monitoreo sin contacto directo con el paciente que mida el patrón respiratorio y la extracción de frecuencia respiratoria es el punto clave para el éxito del proyecto. El sistema presentado en [9] consta de una cámara RGB incorporada en una computadora portátil y un algoritmo para el pos-procesamiento de datos de vídeo adquiridos. A partir del registro de los movimientos del pecho de un sujeto, el análisis de los cambios de intensidad de los píxeles produce una forma de onda que indica un patrón respiratorio.

El asma es una enfermedad causada por la inflamación de las vías aéreas que puede provocar una sensación de ahogo, lo que la posiciona como una enfermedad que puede poner en riesgo la salud de quien la padece. Detectar una crisis de forma oportuna permite al paciente poner en marcha un plan de acción creado por su médico para poder afrontar dicha crisis. La solución propuesta en [4], está basada en un sistema no invasivo para el monitoreo de los ataques de asma, que permite al paciente utilizarlo con ayuda de un familiar o un asistente médico, en el momento que tenga una crisis para llevar un control de la severidad que está presentando la enfermedad.

La Enfermedad Pulmonar Obstructiva Crónica (EPOC) requiere de un control respiratorio y monitoreo de la hipercapnia, es decir, que la sangre del paciente tiene demasiado dióxido de carbono $\left(\mathrm{CO}_{2}\right)$, la cual se produce por no poder respirar adecuadamente. El proyecto reportado en [5] analiza los patrones musculares y respiratorios más característicos de la hipercapnia con el propósito de predecir y simular apropiadamente la respuesta a estos estímulos.

Con el conocimiento obtenido al revisar la literatura, se llegó a la conclusión de que es factible crear un sistema como el propuesto y se procedió con las siguientes fases del proyecto.

\section{Fase actual de la investigación}

La primera fase de este proyecto fue realizar una revisión de literatura para conocer los avances actuales en el área.

La fase dos consintió en investigar y conocer distintos tipos de sensores utilizados para el monitoreo de la respiración, con base en este análisis se concluyó que, para obtener los datos de la frecuencia de respiración del paciente, se utilizará el sensor respiratorio XeThru X4M200, mismo que ya programado, enviará la información del paciente al modelo de Inteligencia Artificial que se encargará de predecir haciendo uso de los datos obtenidos.

La fase en la que se encuentra el proyecto es el diseño y desarrollo del primer prototipo de sistema vestible IoMT para el monitoreo remoto de la respiración, que, con uso de una aplicación móvil, el familiar o el cuidador del paciente, podrá monitorear de manera remota los resultados que el dispositivo genere en tiempo real. Para generar el contexto de uso del hardware y software se está utilizando el diseño participativo [2] con adultos mayores y familiares, por cuestiones de la contingencia sanitaria este proceso ha sido complicado pero se espera quede cubierto en el presente semestre escolar. Las etapas que se seguirán son: entrevistas, codiseño (card-sorting, mapa de ecosistema y lluvia de ideas) y una etapa de validación por expertos de la propuesta,

La siguiente fase será el entrenamiento de un modelo de ML que sea capaz de detectar patrones anormales de respiración los cuales pueden generar riesgos a la salud de acuerdo con la literatura científica del área.

Finalmente se realizará una evaluación de la aceptación tecnológica del prototipo desarrollado en este proyecto.

La Figura 1 muestra la arquitectura propuesta del sistema vestible IoMT.

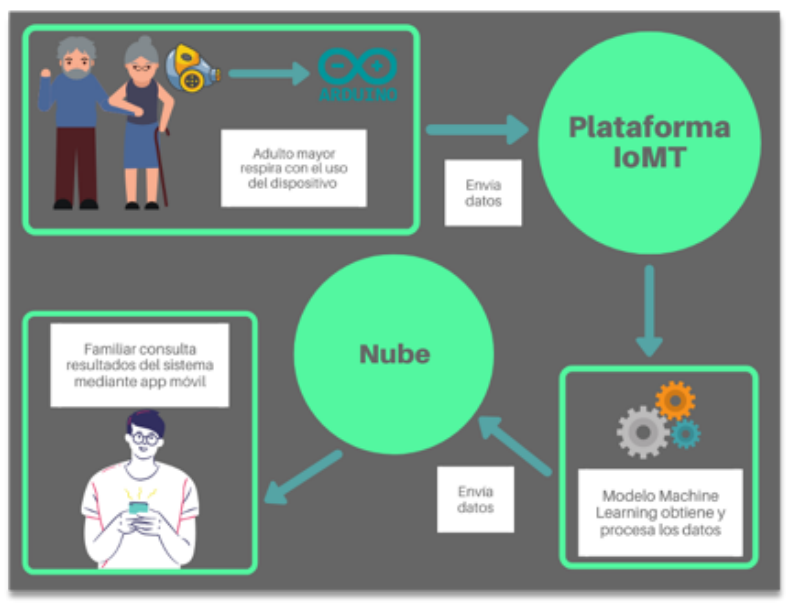

Figura 1. Arquitectura propuesta del sistema.

\section{Contribuciones esperadas y retos de la investigación}

El presente proyecto tiene como objetivo lograr las siguientes contribuciones:

- Entendimiento del contexto de adultos mayores propensos a padecer enfermedades de las vías respiratorias.

- Reporte técnico sobre los distintos sensores utilizados para el monitoreo de la respiración. 
- Modelo de ML para detectar patrones anormales de respiración que puedan generar riesgos a la salud de acuerdo con la literatura científica del área.

- Un prototipo de un sistema vestible IoMT con capacidad de monitoreo remoto de la respiración en adultos mayores que sea capaz de realizar predicciones de posibles situaciones de riesgo de salud en adultos mayores por medio de métodos de aprendizaje automático.

- Reporte de la evaluación con adultos mayores para conocer la aceptación que tienen para el uso del prototipo desarrollado.

\section{Motivación para asistir al consorcio de posgrado}

La asistencia al Consorcio de Posgrado de MexIHC 2021 encajaría muy bien en este trabajo, ya que la guía de reconocidos expertos ayudaría a avanzar en el proyecto, ampliar el aprendizaje y mejorar este proyecto. Además, esta participación podría ayudar a ampliar mis conexiones de red para colaborar en futuros proyectos de investigación con otros estudiantes de posgrado y futuros investigadores.

\section{Referencias}

[1] José Fernando Arboleda. 2016. Sistema para el apoyo a la atención domiciliaria mediante redes de sensores inteligentes. Retrieved from http://bibliotecadigital.udea.edu.co/dspace/bitstream/10 495/7402/1/ArboledaJose_2016_SistemaAtencionDomi ciliaria.pdf

[2] Christian Dindler, Rachel Smith, and Ole Sejer Iversen. 2020. Computational empowerment: participatory design in education. CoDesign 16, 1: 66-80.

[3] Araceli Escobar-Rojas, Jonathan Castillo-Pedroza, Cruz Cruz-Hervert, and Renata Báez-Saldaña. 2015.

Tendencias de morbilidad y mortalidad por neumonía en adultos mexicanos (1984-2010). Neumología y cirugía de tórax 74 .

[4] J. A. González-Godínez and M. A. Farrera-GrajalesSantiago. 2017. Prototipo de camisa monitoreo a pacientes con asma y control clínico. Memorias del Congreso Nacional de Ingeniería Biomédica 4, 1: 393 396.
[5] A M Hernádez, M A Mañanas, R Rabinovich, S Benito, and P Caminal. 2004. Obtención y predicción del patrón respiratorio mediante modelado de su sistema de control ante estímulos hipercápnicos. Memorias de las XXV Jornadas de Automática.

[6] José Luis Ibarra Cornejo, María José Fernández Lara, Elena Viviana Aguas Alveal, Alex Felipe Pozo Castro, Bárbara Antillanca Hernández, and Diego Galvarino Quidequeo Reffers. 2018. Efectos del reposo prolongado en adultos mayores hospitalizados. Anales de la Facultad de Medicina 78, 4: 439.

[7] Juárez-Ramírez, Clara, Márquez-Serrano, Margarita, Salgado de Snyder, Nelly, Pelcastre-Villafuerte, Blanca Estela, Ruelas-González, María Guadalupe, and ReyesMorales Hortensia. 2014. La desigualdad en salud de grupos vulnerables de México: adultos mayores, indígenas y migrantes. Revista panamericana de salud pública 35 .

[8] Noemi Luque and Maicol Ortega. 2020. Análisis de sistemas para registros médicos electrónicos en clínicas y su enfoque al Machine Learning. Retrieved from http://repositorio.ucsp.edu.pe/bitstream/20.500.12590/1 6206/4/LUQUE_SUCASAIRE_NOE_MAC.pdf.

[9] Carlo Massaroni, Daniela Lo Presti, Domenico Formica, Sergio Silvestri, and Emiliano Schena. 2019. NonContact Monitoring of Breathing Pattern and Respiratory Rate via RGB Signal Measurement. Sensors 19, 12: 2758.

[10] P Rojas, V Díaz, P Sacramento, M Rodríguez, R Martínez, and P Delgado. 2016. Mortalidad por enfermedades respiratorias en el adulto mayor. Evolución en un año. Acta Médica del Centro 10. Retrieved from https://www.medigraphic.com/pdfs/medicadelcentro/me c-2016/mec163e.pdf.

[11] Pedro C. Santana-Mancilla, Luis E. Anido-Rifón, Juan Contreras-Castillo, and Raymundo BuenrostroMariscal. 2020. Heuristic Evaluation of an IoMT System for Remote Health Monitoring in Senior Care. International Journal of Environmental Research and Public Health 17, 5: 1586.

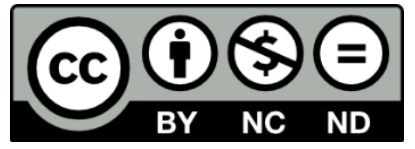

C 2021 by the authors. This work is licensed under the Creative Commons AttributionNonCommercial-NoDerivatives 4.0 International License. To view a copy of this license, visit http://creativecommons.org/licenses/by-nc-nd/4.0/ or send a letter to Creative Commons, PO Box 1866, Mountain View, CA 94042, USA. 